\title{
Beam Patterns of the Five-hundred-metre Aperture Spherical Telescope
}

\author{
B. Dong ${ }^{1,2,3}$ and J. L. $\operatorname{Han}^{1}$ \\ ${ }^{1}$ National Astronomical Observatories, CAS, Jia-20 DaTun Road, Chaoyang District, Beijing 100012, People's Republic of China \\ ${ }^{2}$ School of Physics, University of Chinese Academy of Sciences, Beijing 100049, People's Republic of China \\ ${ }^{3}$ Corresponding author. Email: dongbin@nao.cas.cn
}

(Received December 1, 2012; Accepted January 30, 2013; Online Publication April 24, 2013)

\begin{abstract}
The Five-hundred-metre Aperture Spherical Telescope (FAST) is being constructed in China. With an illuminated aperture of 300-m diameter, it will be the most sensitive single-dish radio telescope in the world. We calculate the beam patterns, gains, and efficiencies of the FAST at $200 \mathrm{MHz}, 1.4 \mathrm{GHz}$, and $3 \mathrm{GHz}$. A program is developed to calculate the structural parameters and construct the FAST models. The three-dimensional beam patterns are calculated by utilising the shooting and bouncing ray method. We show that, with a coaxial horn feed, the FAST has a beam pattern of high gain and reasonably low first sidelobe over the frequency range of $200 \mathrm{MHz}$ to $3 \mathrm{GHz}$. Compared with an ideal 300-m parabolic reflector, the un-illuminated spherical part of the FAST would make the power level near both sides of the main beam rise by at least $20 \mathrm{~dB}$ and the efficiency tends to decrease at high frequencies. At a zenith angle of $0^{\circ}$, its efficiencies at $200 \mathrm{MHz}, 1.4 \mathrm{GHz}$, and $3 \mathrm{GHz}$ are $71.72 \%, 66.94 \%$, and $57.55 \%$, respectively. We conclude that the FAST is an excellent telescope at low frequencies. At high frequencies, the triangular spherical panels and the gaps between panels are important factors that affect the performance of the FAST.
\end{abstract}

Keywords: techniques: miscellaneous - telescopes

\section{INTRODUCTION}

The Five-hundred-metre Aperture Spherical Telescope (FAST) is one of the mega science facilities for basic research in China (Nan et al. 2011). The FAST is being constructed in a karst depression in the Guizhou province at a geographical latitude of about $+26^{\circ}$. It is planned to be completed around 2016. The FAST will be about 10 times more sensitive than the fully steerable 100-m telescopes, e.g. the Effelsberg 100$\mathrm{m}$ telescope and the Green Bank 100-m Telescope (GBT), and about three times as sensitive as the Arecibo telescope. The main reflector of the FAST consists of about 4,400 active panels; the illuminated panels are adaptive to form a $300-\mathrm{m}$ diameter paraboloid when observing (Qiu 1998).

The performance of a radio telescope depends on the beam pattern, which is characterised by gain, beam shape, levels of the first sidelobe, and far-out sidelobes. Poor beam patterns and high sidelobes result from the surface aberration, the blockage of the cabin and the support structure, which introduce additional system temperature from the ground and other radio sources. The sidelobes of the telescope can make observed intensity of 21-cm line variable, and even distant sidelobes can contribute to Stokes $V$ of $21-\mathrm{cm}$ line profiles.
The knowledge of sidelobe responses is very necessary for further corrections (e.g. Robishaw \& Heiles 2009). Arecibo has a squint and squash main beam and highly polarised sidelobes because of blockage (Heiles et al. 2001). High efficiency is generally attempted when a radio telescope is built. In general, the efficiency of a telescope should be higher than $50 \%$. The GBT can achieve an efficiency better than $70 \%$ up to $20 \mathrm{GHz}$ because it has no blockage in optics. ${ }^{1}$ The Effelsberg 100-m telescope can obtain an efficiency higher than $60 \%$ up to $20 \mathrm{GHz}$ at an elevation of $30^{\circ}$ and with a primaryfocus feed. ${ }^{2}$ In some astronomical observations, such as the C-Band All-Sky Survey (C-BASS), the telescope is designed to restrict the far-out sidelobes to be as low as $-70 \mathrm{~dB}$ (Holler et al. 2011).

Because of the huge physical size and complicated structure, the beam performance of the FAST is difficult to calculate. We have developed a program to calculate the structural parameters of the FAST and construct the FAST beam

\footnotetext{
${ }^{1}$ http://www.gb.nrao.edu/ rmaddale/GBT/ReceiverPerformance/ PlanningObservations.htm

${ }^{2}$ http://www.radionet-eu.org/rnwiki/UpgradingtheHorizonPresentations? action $=$ AttachFile\&do= get\&target $=$ keller.pdf
} 
Table 1. Main specifications of the FAST.

\begin{tabular}{ll}
\hline \hline Aperture of the main reflector & $500 \mathrm{~m}$ \\
Radius of the main reflector & $300 \mathrm{~m}$ \\
Opening angle & $112.8^{\circ}$ \\
Illuminated aperture & $300 \mathrm{~m}$ \\
Focal ratio & $f / D=0.4665$ \\
Sky coverage & $z<40^{\circ}$ \\
Frequency coverage & $0.07-3 \mathrm{GHz}$ \\
Sensitivity ( $L$ band) & $A / T \sim 2000$ \\
Resolution $(L$ band) & $2.9 \mathrm{arcmin}$ \\
Slewing time & $<10 \mathrm{~min}$ \\
Pointing accuracy & $8 \mathrm{arcsec}$ \\
\hline \hline
\end{tabular}

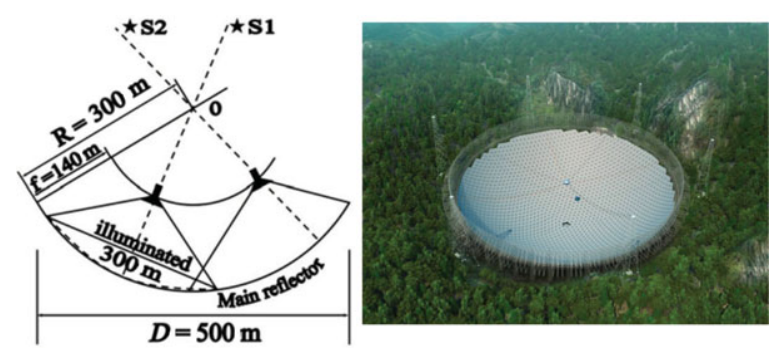

Figure 1. FAST optical geometry (left) (Nan 2006) and its 3D model (right) (Nan et al. 2011).

models. The aim of this paper is to assess the beam performance of the FAST before it is built. In Section 2, we briefly describe the general specifications and our models for the FAST at different zenith angles of $0^{\circ}, 27^{\circ}$, and $40^{\circ}$ and at $200 \mathrm{MHz}, 1.4 \mathrm{GHz}$, and $3 \mathrm{GHz}$. Section 3 shows the calculation results of the FAST beams with brief discussions. Section 4 summarises this work.

\section{GENERAL SPECIFICATIONS AND MODELS OF THE FAST}

Figure 1 shows the optical geometry (Nan 2006) and a threedimensional (3D) model image of the FAST (Nan et al. 2011). The terrain in Figure 1 is an imitation of the real depression around the FAST. The overall diameter of the FAST is 500 $\mathrm{m}$. The FAST consists of about 4400 active reflector panels; each panel has a triangular shape with a spherical surface of a curvature radius of $318.5 \mathrm{~m}$ (Nan et al. 2011). These panels sit on a cable net in a spherical shape with a 300$\mathrm{m}$ radius. The focus cabin is suspended about $140 \mathrm{~m}$ above the reflector. During the observation, illuminated panels of the main reflector will be adjusted instantaneously to form a 300-m paraboloid under the real-time control. The optics of the FAST allows the usage of normal standard feeds. The FAST will work in a frequency range from $70 \mathrm{MHz}$ to $3 \mathrm{GHz}$ in the first construction stage, and be able to observe up to a zenith angle of $40^{\circ}$. The main specifications of the FAST are listed in Table 1.

To calculate the FAST beams at $200 \mathrm{MHz}, 1.4 \mathrm{GHz}$, and 3 GHz to show the performance of the FAST over its operating frequency range, the models we use for the FAST mainly

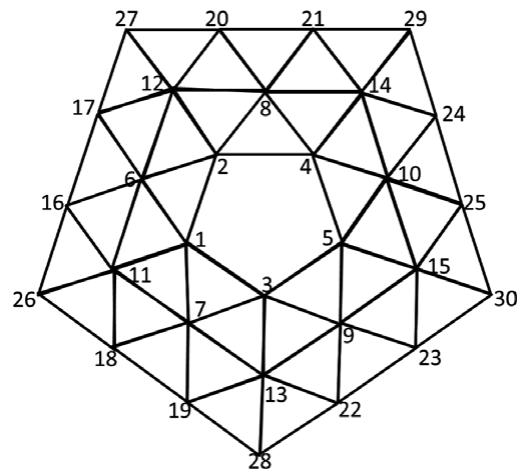

Figure 2. Panels and control nodes in the most central region of the FAST.

involve two components: (1) a feed and (2) the main reflector of 4,400 triangular spherical panels (Figure 2) which are assumed to be perfect electrical conductor with no thickness. The feed we use for the models is a universal coaxial horn with seven corrugated walls (Figure 3). This coaxial feed has good symmetrical patterns, very low level sidelobes and low level cross-polarisation of better than $-39 \mathrm{~dB}$ within the illumination angle. This feed is dual-linearly polarised and illuminates the $300-\mathrm{m}$ paraboloid region of the FAST with an edge taper of about $-10.7 \mathrm{~dB}$ at $\theta=56.4^{\circ}$. This edge taper level is designed as a compromise for the antenna gain and system temperature. As shown in the next section, the efficiency of an ideal 300-m paraboloid is always about $76 \%$, very close to the theoretical maximum value of $\sim 80 \%$ for a primary-focus reflector with a subtended angle of $56.4^{\circ}$ illuminated by a feed with an edge taper of about $-9 \mathrm{~dB}$. A slightly reduced edge taper level of $-10.7 \mathrm{~dB}$ can reduce radiation leakage from the feed to the ground and improve the system temperature. This is especially important for the FAST when the feed illuminates the edge part of the FAST for observations at a zenith angle of $27^{\circ}<z<40^{\circ}$. We assume that the physical size of the feed can be scaled for any frequency, so that the FAST panels could be illuminated by almost the same radiation patterns at any frequency.

Every six panels of the main reflector of the FAST share one common control node, as shown in Figure 2, which is adjusted by an actuator for altitude. There is a gap of about 5 -cm-wide ${ }^{3}$ between every adjacent panels, which is important for the mechanical transformation of the illuminated panels to be adapted to a 300-m paraboloid. Compared with a conventional parabolic radio telescope, the performance of the FAST is mainly limited by three factors. First, the unilluminated region of the FAST tends to create or increase the sidelobes near the main beam. Second, the spherical shape of each panel would be equivalent to surface aberration, which makes the gain decrease more obviously at higher frequencies. Third, the gaps between panels result in leakage and reduce the efficiency of the FAST, especially when the observation wavelength is comparable with the gap size.

\footnotetext{
${ }^{3}$ It is taken from talks of the FAST team and could not be found in any FAST publication.
} 


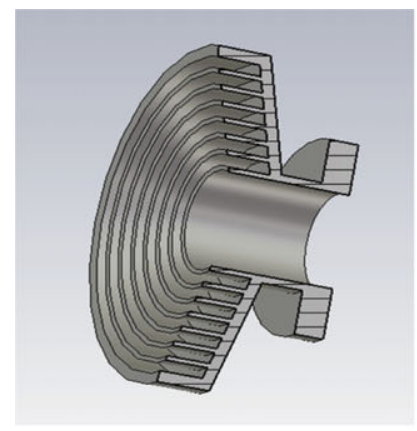

(a)

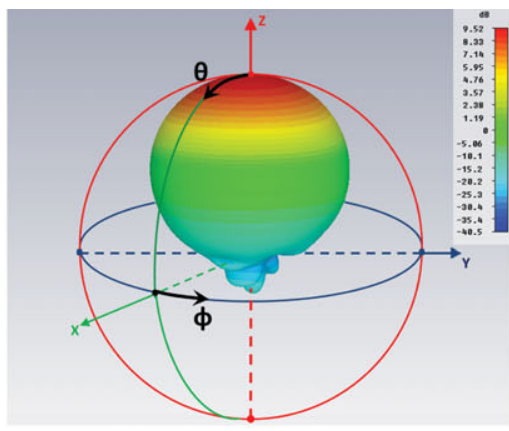

(b)

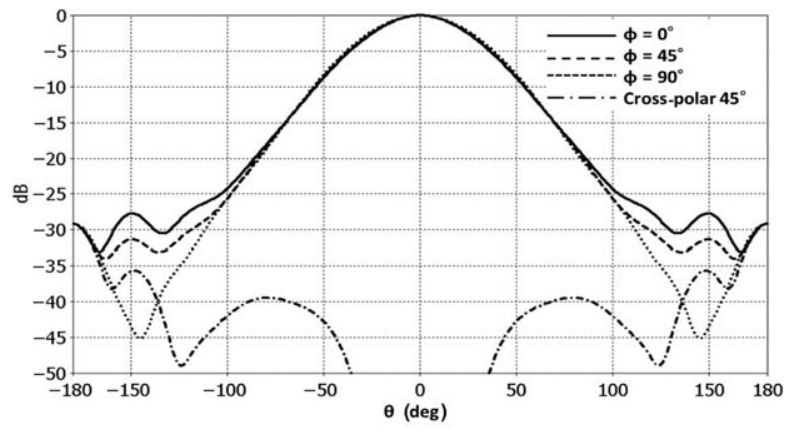

(c)

Figure 3. Coaxial feed (a) 3D cross-sectional view; (b) 3D radiation pattern; (c) cuts of the radiation pattern in the plane of $\phi=0^{\circ}, 45^{\circ}$, and $90^{\circ}$; the low cross-polarisation is also shown.
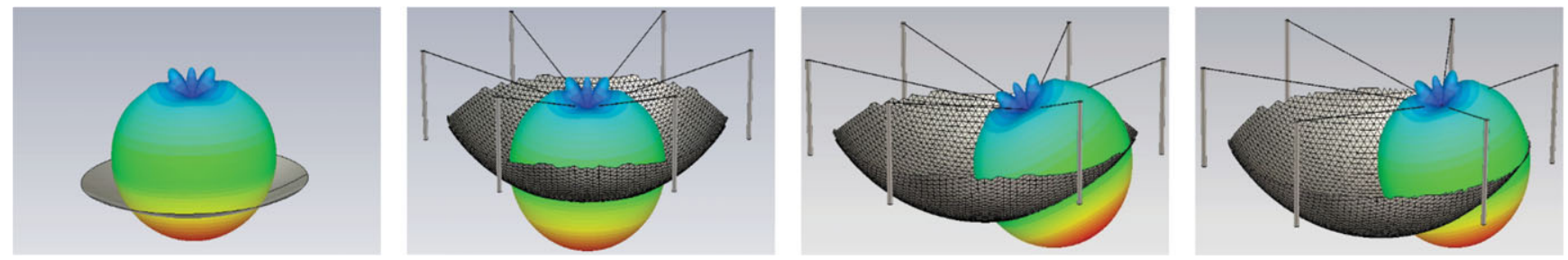

Figure 4. Feed illumination to an ideal 300-m paraboloid and the three FAST models for observations at $z=0^{\circ}, 27^{\circ}$, and $40^{\circ}$.

We construct three models for the FAST and calculate their beams for the observations at zenith angles $z=0^{\circ}$, $27^{\circ}$, and $40^{\circ}$ (see Figure 4), which correspond to 'a central paraboloid of $300 \mathrm{~m}$ ', 'an edge paraboloid of $300 \mathrm{~m}$ ' for observations of $S 1$ in Figure 1, and 'an edge paraboloid of $<300$ m' for observations of $S 2$. In addition, the feed cabin, six support towers, and the cables are roughly modeled in the beam calculations.

\section{CALCULATION RESULTS OF THE FAST BEAMS AND DISCUSSIONS}

We calculate the beam performance of the FAST at observation frequencies of $200 \mathrm{MHz}, 1.4 \mathrm{GHz}$, and $3 \mathrm{GHz}$, respectively, from all panels using the shooting and bouncing ray (SBR) method, which is an extension of physical optics and is capable of simulating a telescope with an electrical size of many thousands of wavelengths. For comparison and verification of our program, we first calculated the beams at the three frequencies for an ideal 300-m parabolic telescope with the same feed at the primary focus.

\subsection{The beams of an ideal 300-m parabolic telescope}

An ideal model for the 300-m parabolic telescope has a coaxial feed at the primary focus. No support structures are involved in the calculation for the clean beams at $200 \mathrm{MHz}$, $1.4 \mathrm{GHz}$, and $3 \mathrm{GHz}$. The 3D beam patterns at the three frequencies are shown in the first row of Figure 5, 2D colored patterns in the second row, the cuts of beams in $\phi=0^{\circ}, 45^{\circ}$, and $90^{\circ}$ planes for far sidelobes and for near sidelobes are shown in the last two rows.

An ideal 300-m parabolic telescope has an excellent performance. It has a high gain of $54.77,71.67$, and $78.28 \mathrm{dBi}$ at the three frequencies. The beams are very symmetrical and have a low-level cross-polarisation of $\sim-50 \mathrm{~dB}$. The first sidelobe is $\sim-30 \mathrm{~dB}$ and the half-power beamwidth (HPBW) is 20.7, 2.97, and 1.41 arcmin at three frequencies.

An ideal 300-m parabolic telescope has a consistent beam performance, with a stable efficiency of $76 \%$ at all frequencies, very close to the theoretical maximum efficiency for a typical prime-focus radio telescope with a subtended angle of $56.4^{\circ}$. This makes the ideal $300-\mathrm{m}$ parabolic telescope a good reference for comparison with the FAST beams at different zenith angles.

\subsection{The FAST beams at $200 \mathrm{MHz}$}

The beam patterns of the FAST for 200-MHz observations at zenith angles of $z=0^{\circ}, 27^{\circ}$, and $40^{\circ}$ are shown in Figure 6 . The 3D beam patterns are shown in the first and second rows and colored 2D patterns in the third row. The cuts in different $\phi$ planes are shown in the last two rows. For comparison, in Figure 7, we show the cuts of the beams in the plane of $\phi=45^{\circ}$ for the FAST and for the ideal 300-m parabolic telescope. The beam patterns for the far sidelobes within $\theta=-180^{\circ}$ to $180^{\circ}$ are calculated with a step of $\theta=1^{\circ}$ and $\phi=2^{\circ}$. The beam patterns for the near sidelobes within $\theta=-2^{\circ}$ to $2^{\circ}$ are calculated with a step of $\theta=0.01^{\circ}$ and $\phi$ 

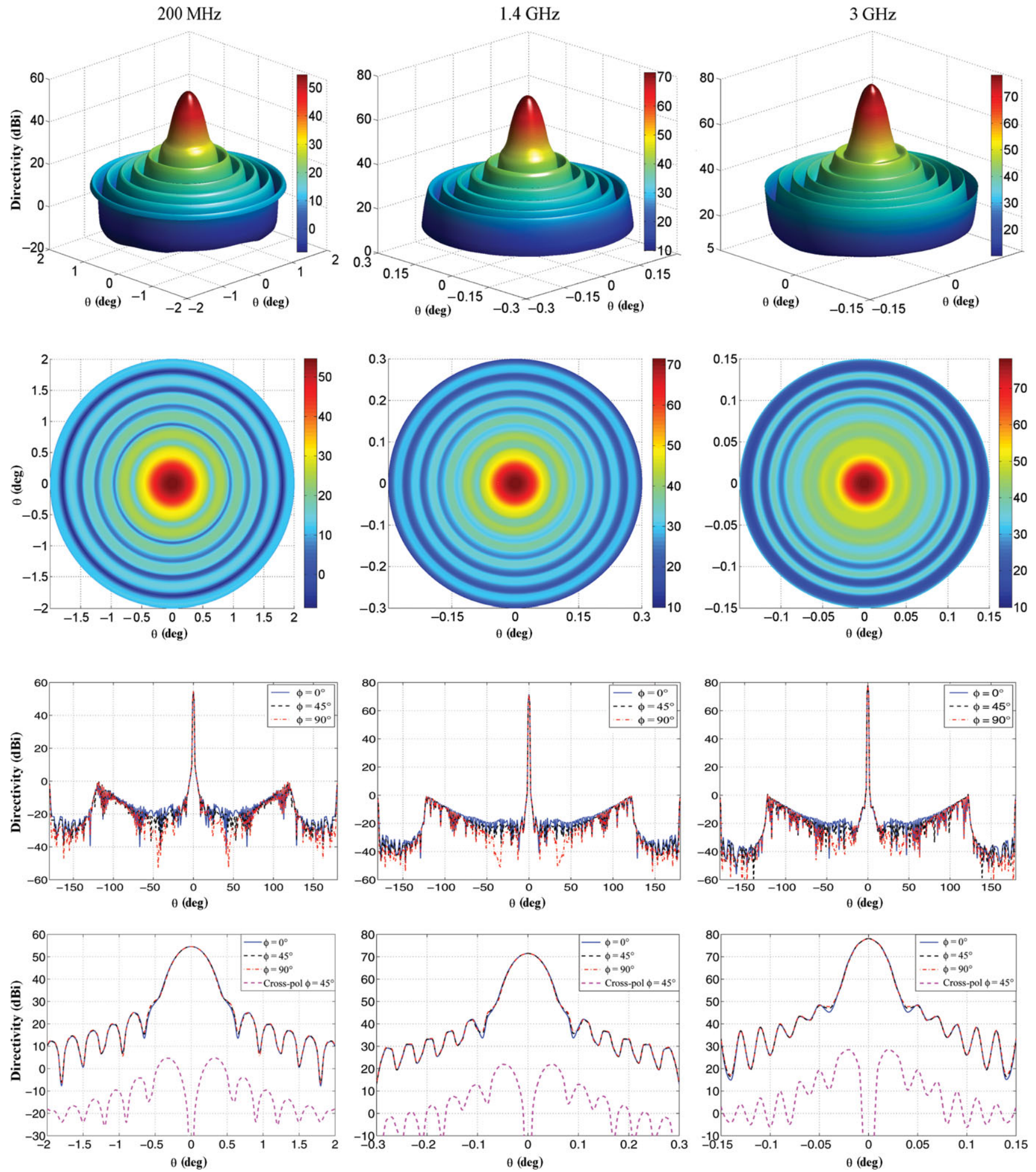

Figure 5. The beam patterns of an ideal $300-\mathrm{m}$ parabolic telescope at $200 \mathrm{MHz}$ (left panels), $1.4 \mathrm{GHz}$ (middle panels), and $3 \mathrm{GHz}$ (right panels). The 3D beams are shown in the first row, the $2 \mathrm{D}$ beam image in the second row, and the cuts in $\phi=0^{\circ}, 45^{\circ}$, and $90^{\circ}$ for the central beam and for far sidelobes in the last two rows.

$=0.5^{\circ}$ (after interpolating). At each observation angle, the calculation time required is about 4 hours on a PC with Interl Core i7-2600 CPU (3.4 GHz) and the memory used is more than $3 \mathrm{~GB}$.
The FAST has very good performance at $200 \mathrm{MHz}$. Compared with an ideal 300-m parabolic telescope, the gain of the FAST shows only a very slight decrease from $54.77 \mathrm{dBi}$ to $54.51 \mathrm{dBi}$ at $z=0^{\circ}$. However, a large number of sidelobes 

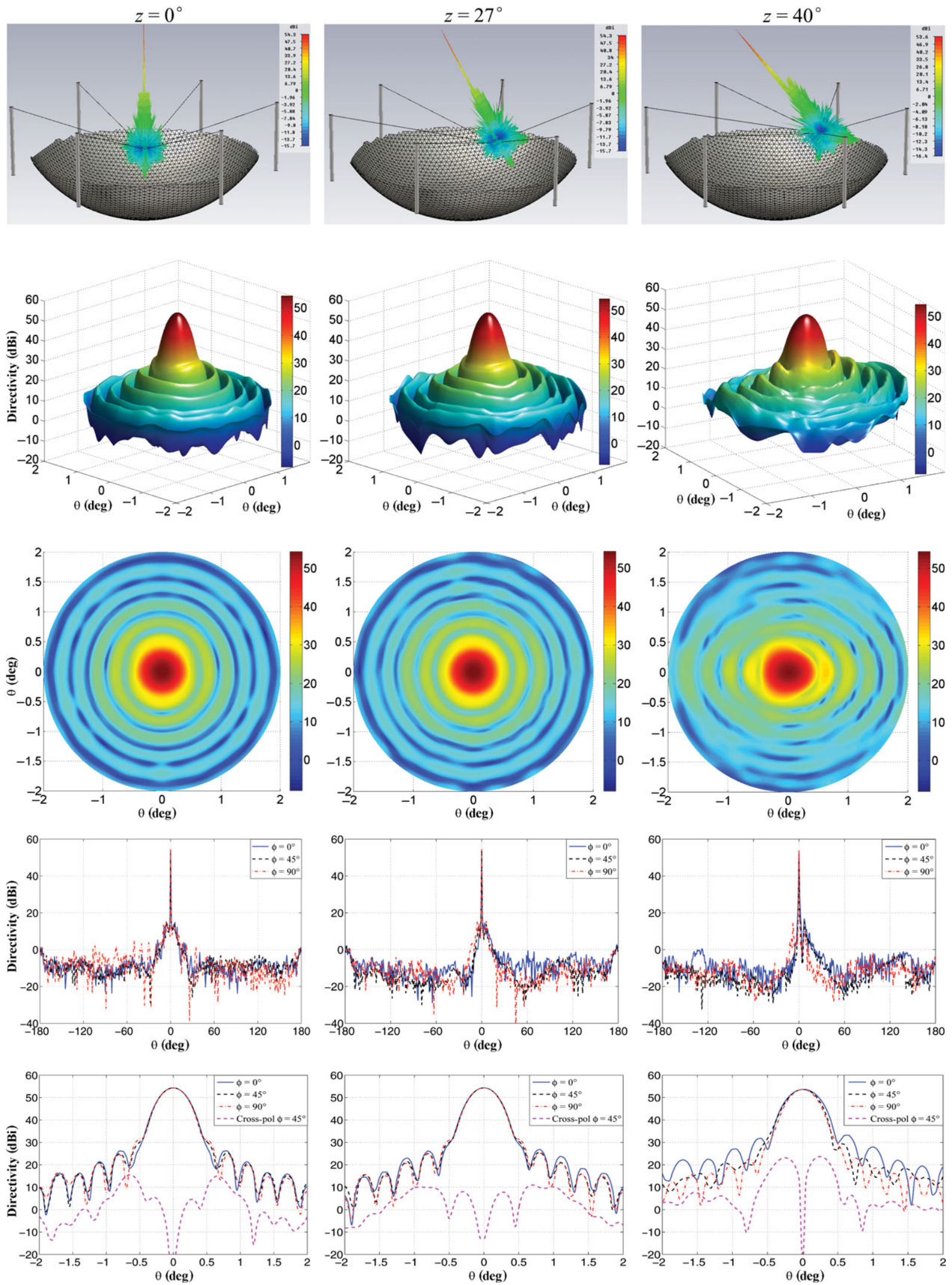

Figure 6. FAST beams for 200-MHz observations at $z=0^{\circ}$ (left panels), $27^{\circ}$ (middle panels), and $40^{\circ}$ (right panels). The full 3D beam patterns are shown in the first row, the 3D patterns for the central part of the beam in the second row, and the 2D beam images in the third row. The cuts of beams in $\phi=0^{\circ}, 45^{\circ}$, and $90^{\circ}$ for the far sidelobes and for the central part of the beam are shown in the last two rows. 

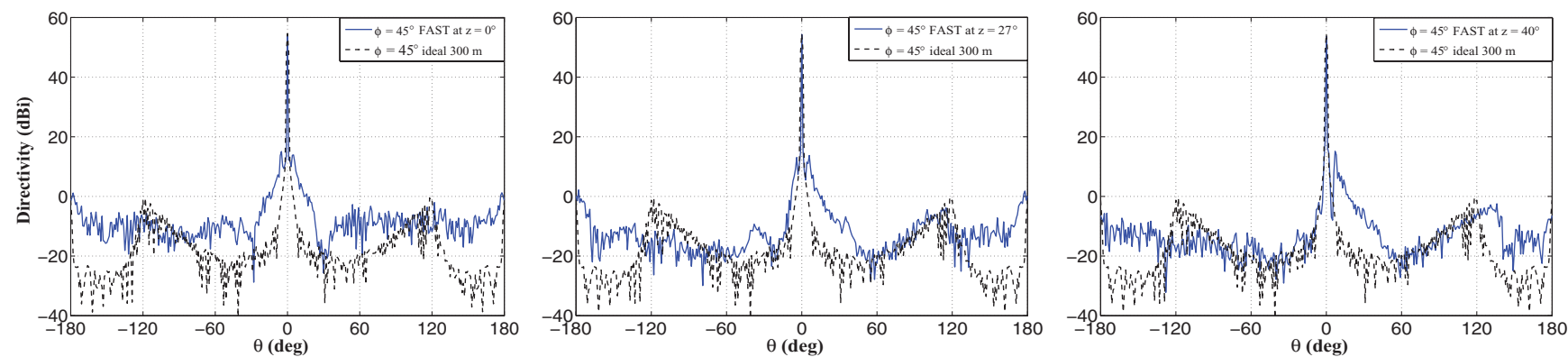

Figure 7. Comparisons of the beam cuts at $200 \mathrm{MHz}$ in the $\phi=45^{\circ}$ plane with the ideal 300-m parabolic telescope at $z=0^{\circ}$ (left), $27^{\circ}$ (middle), and $40^{\circ}$ (right).

(the sharp spikes in the cuts) have emerged in the beam patterns, some as high as $15.5 \mathrm{dBi}$, which are mainly located on both sides of the main beam. These sidelobes are caused by additional reflections from the un-illuminated panels of the FAST and by the non-uniformity of the aperture distribution. The far-out sidelobes have a level of 5-10 dB higher than that of an ideal 300-m parabolic telescope, and the cuts for the far sidelobes vary significantly in different $\phi$ planes. Nevertheless, these sidelobes are still relatively very low compared with the gain of the main beam.

For the FAST observation at $z=27^{\circ}$, the beam performance is much similar to that of $z=0^{\circ}$, except that the power level on the right shoulder of the main beam is obviously higher than that on the left side, which indicates that the sidelobe is caused mainly by the un-illuminated panels of the FAST. For the convenience of comparison, the 2D patterns have been shifted by $27^{\circ}$ to ensure that the main beam is plotted at $\theta=0^{\circ}$. The 3D patterns around the main beam for both $z=0^{\circ}$ and $z=27^{\circ}$ have been degraded by a similar extent. The first sidelobe rises for about $1-2 \mathrm{~dB}$, and the shape of the sidelobes is not as symmetrical as that of an ideal 300-m paraboloid. However, within first nulls, the main beam keeps good symmetry. The HPBW is 21 arcmin, very close to that of the ideal 300-m paraboloid. The first sidelobe is about $-28 \mathrm{~dB}$ below the peak. This low-level sidelobe is mainly due to the taper level of the feed and the no-blockage design of the FAST.

When observations are carried out at $z=40^{\circ}$, the feed cabin of the FAST would move from the position of $z=$ $27^{\circ}$ to $z=40^{\circ}$ in a circular orbit. However, the mobility of panels is limited by $\pm 47.5 \mathrm{~cm}$ (see Nan et al. 2011), the illuminated aperture of the FAST would obviously decrease at this position, and part of the radiation energy from feed would be leaked to the ground. To solve this problem, the feed cabin rotates from the axis of $z=40^{\circ}$ by an offset angle of $10^{\circ}-20^{\circ}$ to increase the illuminated aperture, and the panels are adjusted accordingly to form a new off-axis paraboloid. In our FAST models, we choose this offset angle of $15^{\circ}$ in order to keep the same illumination taper at the edge of the FAST. The gain of the so-formed FAST beam at $z=40^{\circ}$ then decreases to $53.68 \mathrm{dBi}$ due to the smaller total illuminated aperture than $300 \mathrm{~m}$. Due to a larger un- illuminated area, there is a strong sidelobe of about $17 \mathrm{dBi}$ on the right shoulder of the main beam in the plane of $\phi=$ $0^{\circ}$, higher than that at $z=0^{\circ}$ and $z=27^{\circ}$. Within the main beam, the 2D patterns vary significantly in different $\phi$ planes and the cross-polarisation is deteriorated to $-29.97 \mathrm{~dB}$. The main beam becomes elliptical and the HPBW values vary from 20.34 to 24.15 arcmin in different $\phi$ planes. Moreover, the first sidelobe is $34.24 \mathrm{dBi}$ in the plane of $\phi=0^{\circ}$, only $-20.44 \mathrm{~dB}$ below the main beam, much higher than that of $z=0^{\circ}$ and $z=27^{\circ}$.

A summary of beam characteristics of the FAST at 200 $\mathrm{MHz}$ can be found in Table 2. The 'aperture efficiency' and 'effective diameter' in Table 2 are post-calculated. The aperture efficiency is obtained by

$$
\epsilon_{\text {ap }}=\cot \left(\theta_{0} / 2\right)^{2}\left|\int_{0}^{\theta_{0}} \sqrt{G_{\mathrm{f}}(\theta)} \tan (\theta / 2) d \theta\right|^{2}=G / D_{\max },
$$

where

$$
\begin{gathered}
D_{\max }=(\pi d / \lambda)^{2}, \\
\theta_{0}=2 \arctan (d / 4 f),
\end{gathered}
$$

where $d$ is the aperture diameter of the reflector, $f$ is the focal distance, $\theta_{0}$ is the subtended angle of the reflector antenna, $\lambda$ is the observation wavelength, $G_{f}(\theta)$ is the radiation pattern of the feed, $G$ is the gain, and $D_{\max }$ is the theoretical maximum directivity of a reflector antenna.

The effective illuminated area at $z=40^{\circ}$ is smaller than $300 \mathrm{~m}$, so the efficiency is difficult to calculate. We made an estimation of the effective aperture diameter from the value of the calculated HPBW:

$$
d_{\text {ap }}=1.2 \lambda / \mathrm{HPBW},
$$

and we get $d_{\mathrm{ap}}=278.17 \mathrm{~m}$ for $z=40^{\circ}$ at $200 \mathrm{MHz}$ from the average value of HPBW. We then get the efficiency of the telescope from the gain, by using

$$
\epsilon=G \lambda^{2} /\left(\pi d_{\text {ap }}\right)^{2} \approx 68.75 \% \text {. }
$$

The effective diameter is calculated from

$$
d_{\mathrm{eff}}=d_{\mathrm{ap}} \sqrt{\epsilon_{\mathrm{ap}}} \text {. }
$$



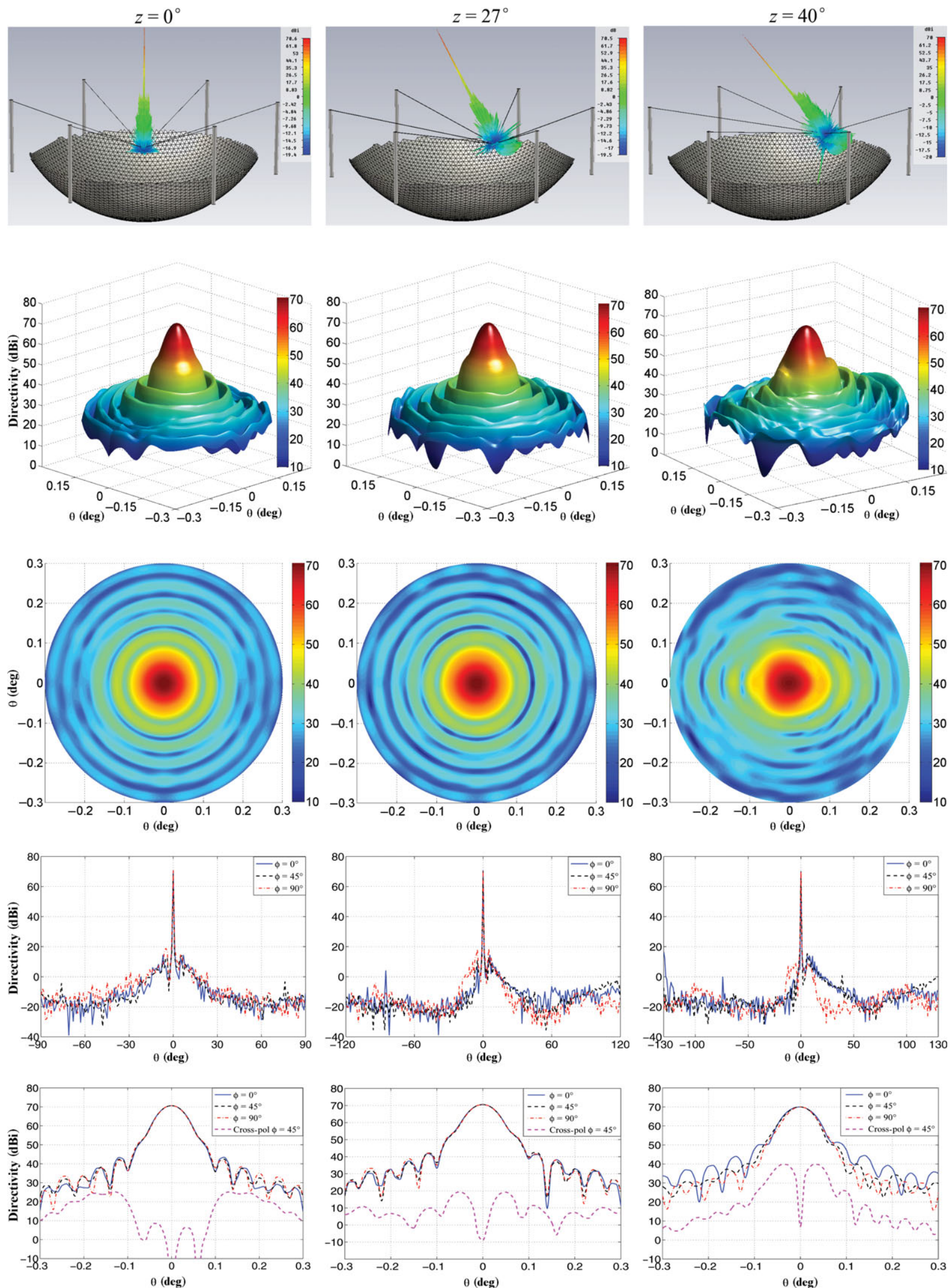

Figure 8. FAST beams at $1.4 \mathrm{GHz}$ for observations at $z=0^{\circ}$ (left panels), $27^{\circ}$ (middle panels), and $40^{\circ}$ (right panels). The full 3D beam patterns are shown in the first row, the 3D patterns for the central part of the beam in the second row, and the 2D beam images in the third row. The cuts of beams in $\phi=0^{\circ}, 45^{\circ}$, and $90^{\circ}$ for the far sidelobes and for the central part of the beam are shown in the last two rows. 
Table 2. Summary of beam characteristics of the FAST at $200 \mathrm{MHz}, 1.4 \mathrm{GHz}$, and $3 \mathrm{GHz}$.

\begin{tabular}{lcccc}
\hline \hline Beam performance & Ideal $300-\mathrm{m} \mathrm{paraboloid}$ & $z=0^{\circ}$ & $z=27^{\circ}$ & $z=40^{\circ}$ \\
\hline & $200 \mathrm{MHz}$ & & & \\
Gain $(G)$ & $54.77 \mathrm{dBi}$ & $54.51 \mathrm{dBi}$ & $54.53 \mathrm{dBi}$ & $53.68 \mathrm{dBi}$ \\
Cross-polarisation (in the $\phi=45^{\circ}$ plane) & $-49.9 \mathrm{~dB}$ & $-38.84 \mathrm{~dB}$ & $-43.35 \mathrm{~dB}$ & $-29.97 \mathrm{~dB}$ \\
First sidelobe level & $-29.53 \mathrm{~dB}$ & $-28.14 \mathrm{~dB}$ & $-27.48 \mathrm{~dB}$ & $-20.44 \mathrm{~dB}$ \\
Power level around the main beam & $-10 \mathrm{dBi}$ & $15.5 \mathrm{dBi}$ & $16 \mathrm{dBi}$ & $17 \mathrm{dBi}$ \\
HPBW (arcmin) & 20.7 & 21 & 21 & $20.34-24.15$ \\
Aperture efficiency $\left(\epsilon_{\text {ap }}\right)$ & $76.07 \%$ & $71.72 \%$ & $71.93 \%$ & $68.75 \%$ \\
Effective diameter $\left(d_{\text {eff }}\right)$ & $261.66 \mathrm{~m}$ & $254.06 \mathrm{~m}$ & $254.43 \mathrm{~m}$ & $230.65 \mathrm{~m}$ \\
& $1.4 \mathrm{GHz}$ & & & \\
Gain $(G)$ & $71.67 \mathrm{dBi}$ & $71.12 \mathrm{dBi}$ & $71.09 \mathrm{dBi}$ & $70.44 \mathrm{dBi}$ \\
Cross-polarisation (in the $\phi=45^{\circ}$ plane) & $-49.56 \mathrm{~dB}$ & $-45.45 \mathrm{~dB}$ & $-51.15 \mathrm{~dB}$ & $-30.14 \mathrm{~dB}$ \\
First sidelobe level & $-29.40 \mathrm{~dB}$ & $-27.23 \mathrm{~dB}$ & $-28.16 \mathrm{~dB}$ & $-18.56 \mathrm{~dB}$ \\
Power level around the main beam & $-8 \mathrm{dBi}$ & $19 \mathrm{dBi}$ & $18 \mathrm{dBi}$ & $16 \mathrm{dBi}$ \\
HPBW (arcmin) & 2.97 & 3.07 & 3.08 & $2.97-3.42$ \\
Aperture efficiency $\left(\epsilon_{\text {ap }}\right)$ & $76.04 \%$ & $66.94 \%$ & $66.44 \%$ & $67.27 \%$ \\
Effective diameter $\left(d_{\text {eff }}\right)$ & $261.60 \mathrm{~m}$ & $245.46 \mathrm{~m}$ & $244.54 \mathrm{~m}$ & $226.94 \mathrm{~m}$ \\
& $3 \mathrm{GHz}$ & & & \\
Gain $(G)$ & $78.28 \mathrm{dBi}$ & $77.10 \mathrm{dBi}$ & $76.96 \mathrm{dBi}$ & $76.05 \mathrm{dBi}$ \\
Cross-polarisation (in the $\phi=45^{\circ}$ plane) & $-49.62 \mathrm{~dB}$ & $-49.25 \mathrm{~dB}$ & $-49.74 \mathrm{~dB}$ & $-29.80 \mathrm{~dB}$ \\
First sidelobe level & $-29.58 \mathrm{~dB}$ & $-34.61 \mathrm{~dB}$ & $-30.16 \mathrm{~dB}$ & $-24.76 \mathrm{~dB}$ \\
Power level around the main beam & $-3 \mathrm{dBi}$ & $18 \mathrm{dBi}$ & $18 \mathrm{dBi}$ & $17 \mathrm{dBi}$ \\
HPBW (arcmin) & 1.41 & $1.48-1.5$ & $1.41-1.44$ & $1.52-1.62$ \\
Aperture efficiency $\left(\epsilon_{\text {ap }}\right)$ & $75.65 \%$ & $57.55 \%$ & $55.82 \%$ & $59.29 \%$ \\
Effective diameter $\left(d_{\text {eff }}\right)$ & $260.92 \mathrm{~m}$ & $227.59 \mathrm{~m}$ & $223.95 \mathrm{~m}$ & $202.07 \mathrm{~m}$ \\
\hline \hline
\end{tabular}
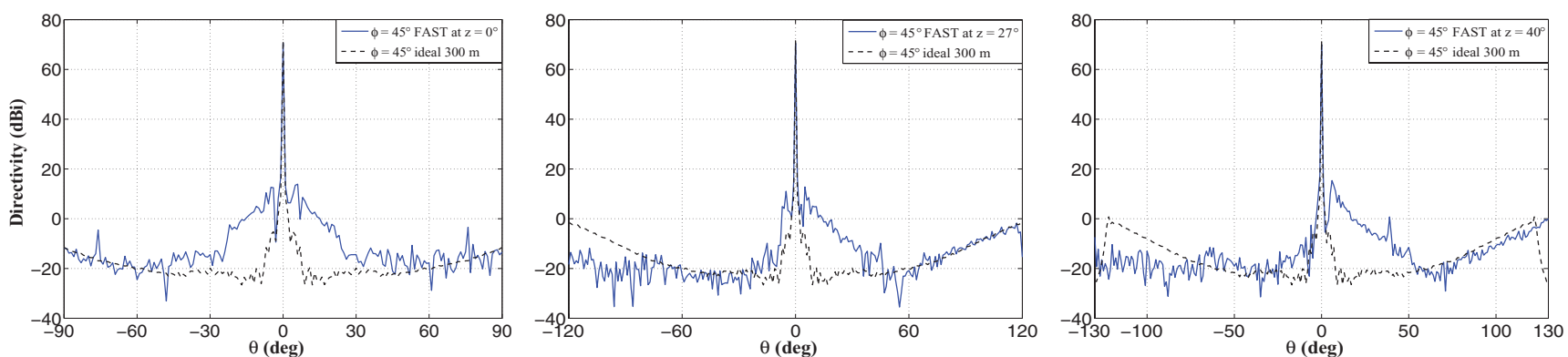

Figure 9. Comparisons of the beam cuts at $1.4 \mathrm{GHz}$ in the $\phi=45^{\circ}$ plane with the ideal $300-\mathrm{m}$ parabolic telescope at $z=0^{\circ}$ (left), $27^{\circ}$ (middle), and $40^{\circ}$ (right).

\subsection{The FAST beams at $1.4 \mathrm{GHz}$}

The beam patterns of the FAST for observations at $1.4 \mathrm{GHz}$ at $z=0^{\circ}, 27^{\circ}$, and $40^{\circ}$ are calculated as shown in Figure 8 . The beam patterns for far sidelobes are calculated with a coarse step of $\theta=1^{\circ}$ and $\phi=5^{\circ}$ because calculations at higher frequencies are very time-consuming. The beam patterns for near sidelobes are calculated within a small range of $\theta=-0.3^{\circ}$ to $0.3^{\circ}$ by a fine step of $\theta=0.001^{\circ}$ and $\phi=0.5^{\circ}$ (after interpolating). For each zenith angle, the calculation time required is about 12 hours on a PC with Interl Core i7-2600 CPU (3.4 GHz) and the memory used is more than 5 GB. For the purpose of reducing calculation time, the range of $\theta$ is restricted to $\theta=-90^{\circ}$ to $90^{\circ}$ for $z=0^{\circ}, \theta=-120^{\circ}$ to $120^{\circ}$ for $z=27^{\circ}$ and $\theta=-130^{\circ}$ to $130^{\circ}$ for $z=40^{\circ}$ since backlobes of the FAST are so small that it can be ignored in our calculation. For comparison, in Figure 9 we show the cuts of the beams in the plane of $\phi=45^{\circ}$ for the FAST and for the ideal 300-m parabolic telescope.

At $1.4 \mathrm{GHz}$, the gain of the FAST beam is up to 71.12 $\mathrm{dBi}$ at $z=0^{\circ}$. Compared with an ideal 300-m parabolic telescope, the gain is decreased by about $0.55 \mathrm{~dB}$. Due to the decreased gain, the aperture efficiency of the FAST decreases to $66.94 \%$, which is equivalent to an effective aperture of $245.46 \mathrm{~m}$ in diameter. A large number of sidelobes (sharp spikes) appear and the power level around the main beam is about $20 \mathrm{dBi}$. The first sidelobe has a level of $43.89 \mathrm{dBi}$, i.e. $-27.23 \mathrm{~dB}$ below peak, similar to that at $200 \mathrm{MHz}$. The HPBW is 3.07 arcmin, and the main beam is very symmetrical in different $\phi$ planes with a low cross-polarisation of $-45.45 \mathrm{~dB}$. The FAST beams at $z=27^{\circ}$ has similar performance with that at $z=0^{\circ}$.

At $z=40^{\circ}$, as expected, the gain of the FAST decreases to $70.44 \mathrm{dBi}$, corresponding to an efficiency of $67.27 \%$ and 
$Z=0^{\circ}$
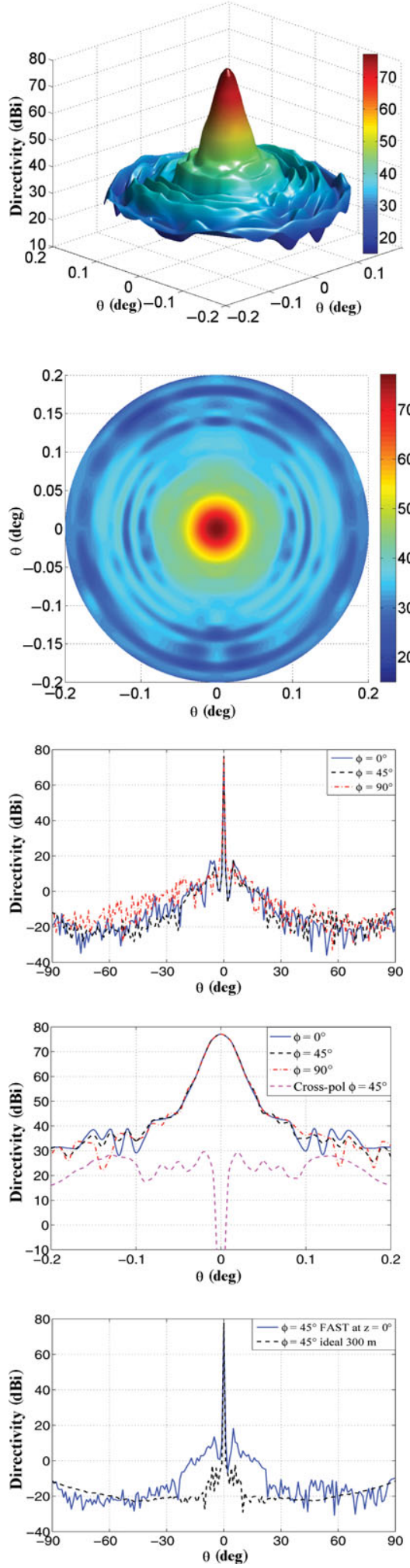

$Z=27^{\circ}$
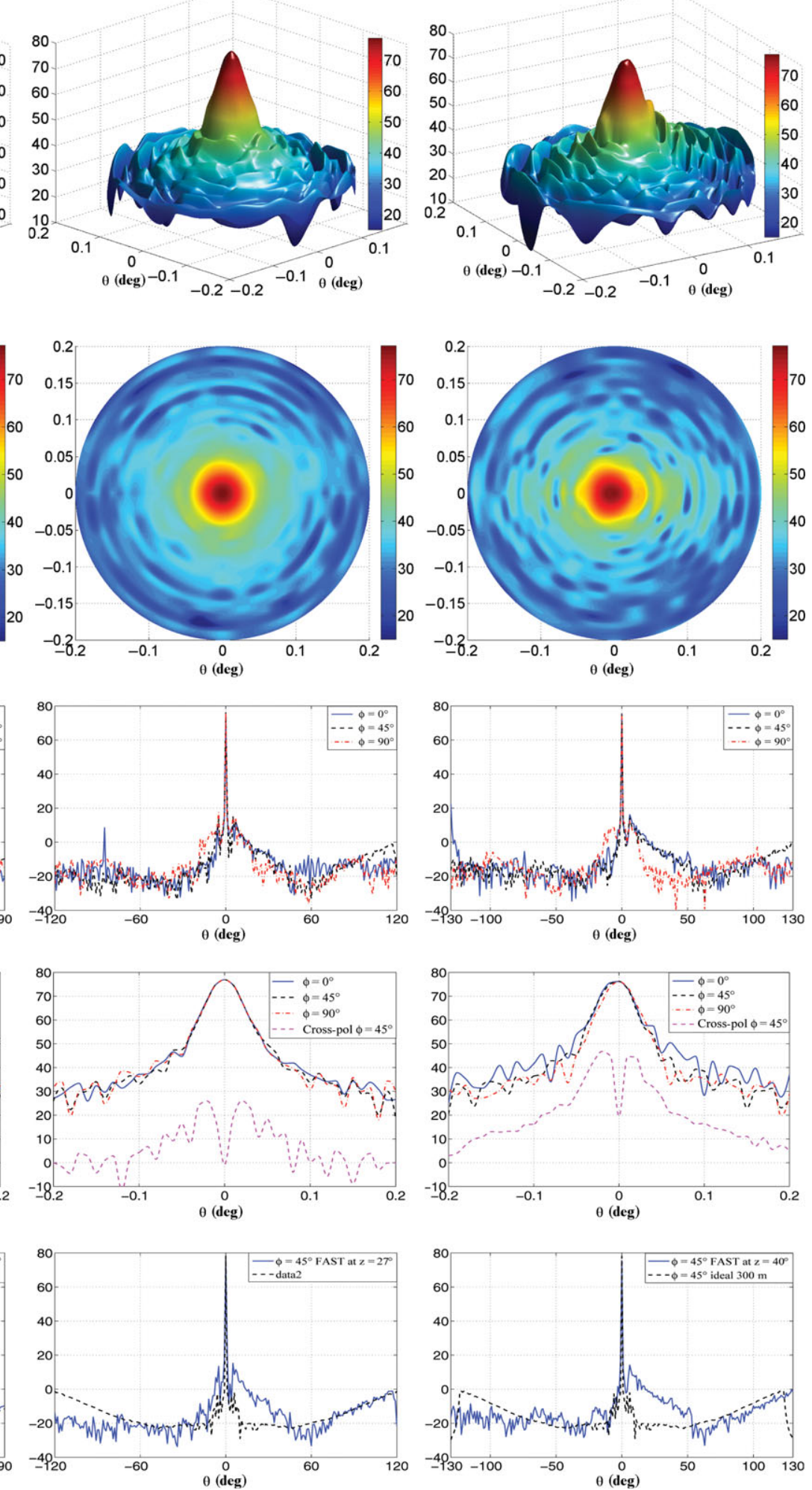

Figure 10. FAST beams at $3 \mathrm{GHz}$ for observations at $z=0^{\circ}$ (left panels), $27^{\circ}$ (middle panels), and $40^{\circ}$ (right panels). The 3D patterns for the central part of the beam are shown in the first row, and the 2D beam images in the second row. The cuts of beams in $\phi=0^{\circ}, 45^{\circ}$, and $90^{\circ}$ for the far sidelobes and for the central part of the beam are shown in the third and fourth rows; comparisons of the cuts in the $\phi=45^{\circ}$ plane with the ideal 300-m parabolic telescope are shown in the last row. 
an effective aperture of $226.94 \mathrm{~m}$. The first sidelobe rises to $51.88 \mathrm{dBi}$ in the plane of $\phi=0^{\circ}$, i.e. $-18.56 \mathrm{~dB}$ below peak. The main beam has an elliptical shape and the HPBW varies from 2.97 arcmin in the plane of $\phi=90^{\circ}$ to 3.42 arcmin in the plane of $\phi=0^{\circ}$.

\subsection{The FAST beams at $3 \mathrm{GHz}$}

The upper frequency limit is $3 \mathrm{GHz}$ for the first construction stage of the FAST. The assessment of beam performance at $3 \mathrm{GHz}$ is very important for the possible development of the FAST to work at $5 \mathrm{GHz}$ or even $8 \mathrm{GHz}$ in the future second stage. However, it is extremely time-consuming to calculate the full 3D beams at $3 \mathrm{GHz}$, probably more than 500 hours for each $z$. We therefore calculate the 2D beam patterns in different $\phi$ planes (see Figure 10). To save time, the simulation range of $\theta$ is restricted to $\theta=-90^{\circ}$ to $90^{\circ}$ for $z$ $=0^{\circ}, \theta=-120^{\circ}$ to $120^{\circ}$ for $z=27^{\circ}$, and $\theta=-130^{\circ}$ to $130^{\circ}$ for $z=40^{\circ}$. The beam patterns for far sidelobes are calculated with a coarse step of $\theta=1^{\circ}$ and the beam patterns for near sidelobes around the main beam are calculated within the range of $\theta=-0.2^{\circ}$ to $0.2^{\circ}$ and with a step of $\theta=0.001^{\circ}$ and $\phi=0.5^{\circ}$ (after interpolating). The calculations of the FAST beams at $3 \mathrm{GHz}$ use a memory of about $8 \mathrm{~GB}$ in our computer.

The gain of the FAST beam at $3 \mathrm{GHz}$ is $77.1 \mathrm{dBi}$ at $z=0^{\circ}$. Compared with an ideal 300-m paraboloid, the gain decreases by $-1.18 \mathrm{~dB}$; this leads to a decrease of aperture efficiency to about $57.55 \%$ and an effective aperture to $227.59 \mathrm{~m}$. The efficiency decrease is serious at $3 \mathrm{GHz}$ because the wavelength of $10 \mathrm{~cm}$ is comparable with the surface abberation of the spherical panels and the gap size. The profile of the main beam is not smooth and the first sidelobe is $-34.61 \mathrm{~dB}$ below the peak. The main beam at $3 \mathrm{GHz}$ is not as symmetrical as that of lower frequencies and the HPBW values vary from $1.48 \operatorname{arcmin}$ (in the plane of $\phi=90^{\circ}$ ) to $1.5 \mathrm{arcmin}$ (in the plane of $\phi=0^{\circ}$ ). The performance of the FAST at $z=27^{\circ}$ is still similar to that at $z=0^{\circ}$.

At $z=40^{\circ}$, the gain of the FAST is $76.05 \mathrm{dBi}$, corresponding to an efficiency of $59.29 \%$ and an effective aperture of $202.07 \mathrm{~m}$. The first sidelobe rises up to $51.29 \mathrm{dBi}$, i.e. only $-24.76 \mathrm{~dB}$ below the main peak. The main beam becomes elliptical with an HPBW of 1.52 arcmin in the plane of $\phi=$ $90^{\circ}$ and 1.62 arcmin in the plane of $\phi=0^{\circ}$.

\section{CONCLUSIONS}

The construction of the FAST telescope began in 2011 and should be completed by late 2016 . Before we work with the FAST, we have to work on the FAST. In this paper, we made models for the FAST and assess the beam performance at $200 \mathrm{MHz}, 1.4 \mathrm{GHz}$, and $3 \mathrm{GHz}$ for future observations at $z=0^{\circ}, 27^{\circ}$, and $40^{\circ}$. We used a scalable coaxial feed with a symmetrical radiation pattern and gain of about $10.5 \mathrm{dBi}$. The telescope beam in fact highly depends on the feed. For example, the first sidelobes in our simulation remain as low as $-30 \mathrm{~dB}$ at all frequencies at $z=0^{\circ}$ and $27^{\circ}$ because of the $-10.7 \mathrm{~dB}$ edge taper of our feed model. For the ideal 300-m parabolic telescope, a higher edge taper (e.g. $-9 \mathrm{~dB})$ of the feed can result in a higher first sidelobe of about $-22 \mathrm{~dB}$ and a slightly higher efficiency of about $78 \%$.

The sidelobes of the FAST beam are mainly located on the shoulders around the main beam, and the power level of the sidelobes around the main beam is about $15-20 \mathrm{dBi}$, much higher than that of an ideal $300-\mathrm{m}$ paraboloid. They are caused by the un-illuminated part of the FAST panels and almost do not vary with frequency. The radio waves coming from the direction of these sidelobes hit the un-illuminated panels and are reflected into the feed, but suppressed by the edge taper level of the feed. The sharp-spikes form of the sidelobes is due to the spherical shape of panels, which reflect the coming radio waves to a focal line rather than to the feed focus.

The spherical shape of panels introduces a surface abberation, which is a major reason for the decreased gain and efficiency of the FAST beams at higher frequencies. Another factor for the decrease of the gain is the gap between panels. With the increase of frequency, the negative effects of the spherical panels and the gap leakage become more significant. At $3 \mathrm{GHz}$, the two factors cause the efficiency of the FAST beam below $60 \%$. The gaps of $5 \mathrm{~cm}$ wide occupy about $1.6 \%$ of the illuminated area. The ground radiation (e.g. $300 \mathrm{~K}$ ) passing through the open gap would cause the system temperature to increase by $300 \mathrm{~K}^{*} 1.6 \%=4.8 \mathrm{~K}$. If the gaps are shielded with soft metal meshes, the increase of system temperature could be much smaller.

Note, however, that our calculations of the FAST beams are made with an ideal model of the feed whose cross polarisation is hard to realise in practice and without consideration of the orthogonal mode transducer, so the cross-polarisation of the FAST beams in Table 2 should be taken as an upper limit. The estimated aperture efficiencies and gains in Table 2 have considered effects of illumination taper, spillover, cross polarisation, phase error, surface aberration of the spherical panels, leakage from the gaps between panels and structure blockage, but not accounted the losses of a practical feed, the orthogonal-mode transducer and deformation of the reflector panels; the effects of edge currents are also not taken into account due to the limit of the SBR method. Therefore, our calculations in this paper are the best values to an ideal extent.

\section{ACKNOWLEDGMENTS}

The authors are supported by the National Nature Science Foundation of China (10833003), and thank CST for support.

\section{REFERENCES}

Heiles, C., et al. 2001, PASP, 113, 1247

Holler, C. M., et al. 2011, IEEE Transactions on Antennas and Propagation, 61, 117

Nan, R. 2006, Sci. China G, 49, 129

Nan, R., et al. 2011, Int. J. Mod. Phys. D, 20, 989

Qiu, Y. H. 1998, MNRAS, 301, 827

Robishaw, T., \& Heiles, C. 2009, PASP, 121, 272 